\title{
Successful reconstruction of ischial pressure sores with inferior gluteal artery descending branch perforator flap
}

\author{
Hanhua Li, Wen Lai, Shaoyi Zheng, Zhifeng Huang, Zuan Liu, Bing Xiong \\ Department of Burns and Wound Repair Surgery, Guangdong Provincial People’s Hospital, Guangdong Academy of Medical Sciences, Guangzhou, \\ China \\ Contributions: (I) Conception and design: H Li, B Xiong; (II) Administrative support: W Lai; (III) Provision of study materials or patients: W Lai, S \\ Zheng, B Xiong; (IV) Collection and assembly of data: H Li, Z Huang, Z Liu; (V) Data analysis and interpretation: H Li; (VI) Manuscript writing: \\ All authors; (VII) Final approval of manuscript: All authors. \\ Correspondence to: Bing Xiong. Guangdong Provincial People's Hospital, Guangdong Academy of Medical Sciences, No. 106, Zhongshan 2 Road, \\ Yuexiu District, Guangzhou, China. Email: drxiongbing@126.com.
}

\begin{abstract}
Background Ischial pressure sores often recur, the surgical choice often troubled the surgeon, because surgery repair should consider future reconstructive procedures. The purpose of this article is to present a new surgical option for the reconstruction of primary or recurrent ischial pressure sores by using an inferior gluteal artery of the descending branch perforator flap.

Methods: A study involving patients suffering from ischial pressure sores was performed from March 2016 to August 2020. Patients with large defects, for which direct closure was not possible, underwent reconstructive surgery using an inferior gluteal artery descending branch perforator flap. Collected data included age, diabetes, wound culture, size of the wound, whether or not negative pressure therapy was received, operative time, intraoperative blood loss, and postoperative complications. Patients were followed up through outpatient visits or by telephone.
\end{abstract}

Results: Five patients with ischial pressure sores (four primary and one recurrent) underwent reconstructive surgery with inferior gluteal artery descending branch perforator flap. One patient received colostomy and wound negative pressure therapy before lesion reconstruction. All patients successfully recovered without postoperative complications. The follow-up period ranged from 4 to 31 months. No wound disruption or recurrence was recorded.

Conclusions: Inferior gluteal artery descending branch perforator flap treatment of ischial pressure sores is a simple and feasible method for preserving the inferior gluteal artery's main vascular perforators and could be used as a future surgical option.

Keywords: Ischial pressure sores; inferior gluteal artery descending branch; perforator flap; reconstruction

Submitted Aug 16, 2020. Accepted for publication Jan 06, 2021.

doi: 10.21037/apm-20-1619

View this article at: http://dx.doi.org/10.21037/apm-20-1619

\section{Introduction}

The ischium is a common site of pressure sores, especially in paraplegic patients who use wheelchairs. The presence of an ischial pressure sore inevitably leads to tissue defects, ischial synovial sac infection, and even osteomyelitis of the ischium. Reconstruction of pressure sore defects is a constant challenge for surgeons. Various procedures have been used to reconstruct ischial pressure sores, with flap coverage being the first choice. There are multiple types of flaps that can be used in flap surgery for pressure sores, each of which has its unique advantages. Examples include the inferior gluteal artery perforator flap (1), internal pudendal artery perforator flap (2), gluteus maximus island 
flap (3), V-Y flap (4), and free flap (5). However, due to the high rates of complications and recurrence, the selection of flaps for reconstructive surgery should consider future reconstructive procedures (6).

The inferior gluteal artery descending branch flap was first used in 1981 to reconstruct the buttock and perineum (7). The flap includes the inferior gluteal and posterior thigh region's subcutaneous skin tissue and the caudal portion of the gluteus Maximus muscle, the inferior gluteal artery, and the posterior cutaneous nerve of the thigh. We found that the posterior thigh presented with several arterial perforators coming from the inferior gluteal artery descending branch. This perforator flap is nourished by the descending branches of the inferior gluteal artery, which has not been reported previously.

In this study, we aimed to report our findings on the use of inferior gluteal artery descending branch perforator flaps to reconstruct ischial pressure sores. In this research, we prepared sufficient high-quality tissues inserted at the acceptor site without necessarily warry the defect of the donor site. We present the following article in accordance with the Case Series reporting checklist (available at http:// dx.doi.org/10.21037/apm-20-1619).

\section{Methods}

A study of patients with ischial pressure sores was performed from March 2016 to August 2020. The study was conducted in accordance with the Declaration of Helsinki (as revised in 2013). The study was approved by the institutional board of Guangdong Provincial People's Hospital, Guangdong Academy of Medical Sciences (No. GDREC2019179H) and informed consent was obtained from all the patients. Patients with large ischial defects underwent reconstruction using the inferior gluteal artery descending branch perforator flap. Patient data were collected, including age, diabetes, wound culture, size of the wound, whether or not negative pressure therapy was received, operative time, intraoperative blood loss, and postoperative complications. The patients were followed up through outpatient visits or by telephone for more than three months.

\section{Statistical analysis}

Statistical analysis was performed using the SPSS 17.0 software. A descriptive analysis was used for data description.

\section{Anatomy of flap design}

Branches of the inferior gluteal artery nourish the skin of the posterior thigh. The inferior gluteal artery originates from the internal iliac artery in the pelvis and passes through the piriformis's lower hole toward the lower border of the gluteus maximus. The artery accompanies the greater sciatic nerve, the internal pudendal vessels, and the posterior femoral cutaneous nerve. In $91 \%$ of cases, branches of the inferior gluteal artery descend into the posterior thigh, accompanied by the posterior femoral cutaneous nerve. Several interconnected perforating arteries distributed along the descending branch nourish the skin of the posterior thigh; even if an artery loses direct blood supply, the skin can still be nourished by the adjacent perforators (8).

\section{Surgical procedure}

Under hard external anesthesia, the patient was placed in a prone position with some hip flexion degree. A slightly oblique skin incision was performed with the long axis parallel to the ischium's anatomical structure. The bursa was infiltrated with methylene blue to aid dissection and ensure complete excision.

Subcutaneous dissection was performed along the sinus of the ischial tuberosity. Complete excision of the bursa was performed deep into the ischial tuberosity, including the ischial tuberosity perichondrium under the wound, exposing freshly oozing bone. The bony stump was flattened to a smooth surface with no spurs or irregularities.

The rotation point was detected under the gluteus maximus by preoperative Doppler. The design was based on the median vertical axis between the ischial tuberosity and the greater trochanter, with the body surface projection of vasculature, parallel to the inferior gluteal artery descending branch. A longitudinal thigh posterior flap was centered on this vertical axis, with the dissection along the deep fascia, thus preserving the cutaneous nerve and avoiding exposing the sciatic nerve. As much of the pedicle flap was retained as possible.

For avoiding tethering and kinking of the pedicle, the flap was rotated into the defect, and torsion was applied evenly, mobilizing the soft tissues around the donor site. A suction drain was inserted under the local flap, and the wound was closed primarily (Figure 1). Postoperatively, patients were placed on a suspension bed to minimize pressure on the flap. 

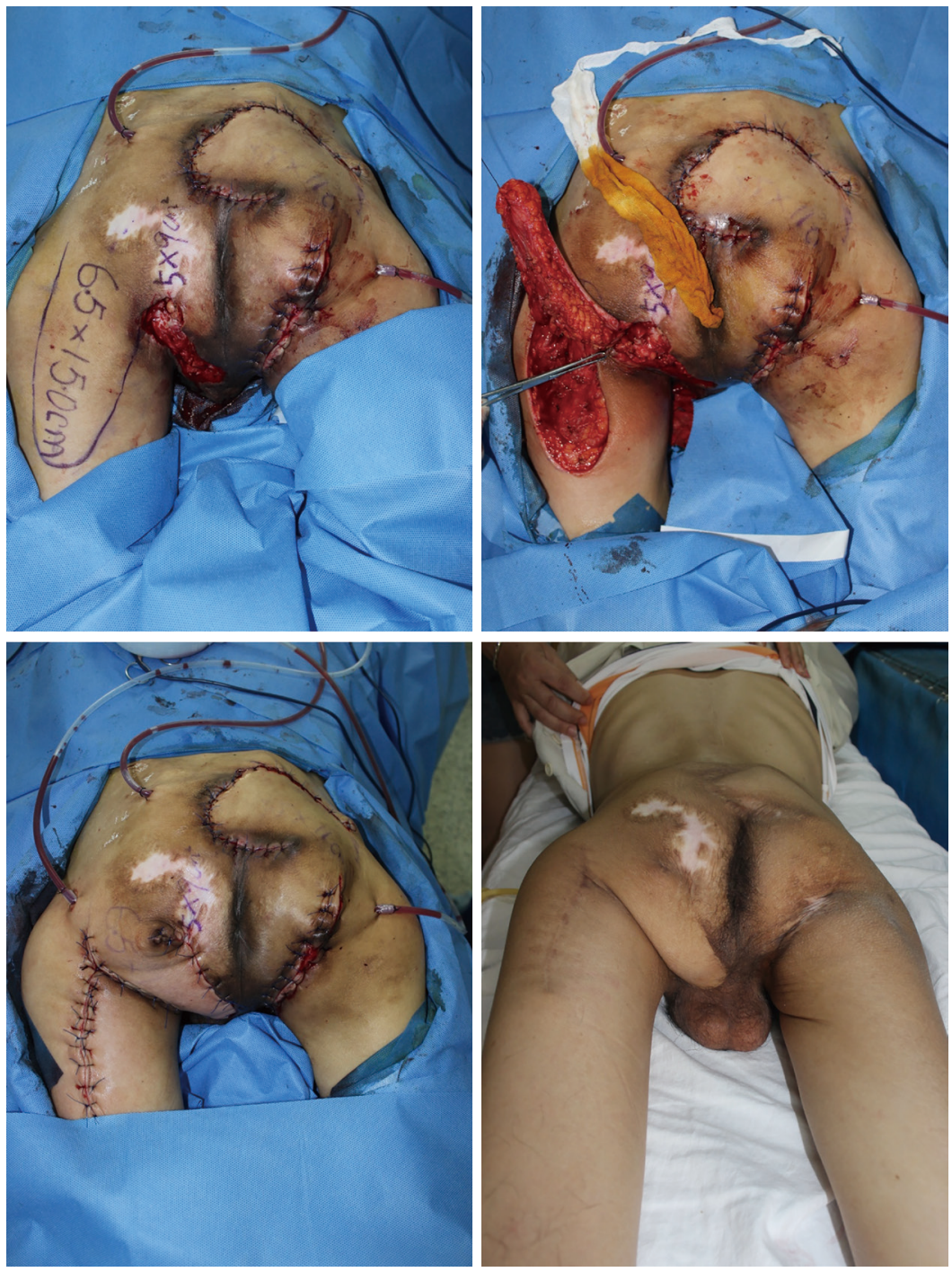

Figure 1 Management of ischial pressure sores with inferior gluteal artery descending branch perforator flap.

\section{Results}

A total of five wounds were reconstructed using the inferior gluteal artery descending branch perforator flap. Four patients were males and one was female, ranging in age from 42 to 73 years old, and each of them had suffered paraplegia due to spinal cord trauma. The time to reconstruction varied from 3 to 8 months after the exposure of ischial pressure ulcers. The sores ranged in size from $6 \mathrm{~cm} \times 5 \mathrm{~cm}$ to $10 \mathrm{~cm} \times 8 \mathrm{~cm}$. One wound was located close to the perineum, and this patient suffered severe malnutrition combined with sepsis and incontinence. The patient underwent colostomy to control the infection; at the same time, he was given negative pressure therapy to assist with wound healing. After ten days of negative pressure therapy, the reconstruction was performed. One patient had a severe wound infection accompanied by ischial osteomyelitis. In this patient, reconstruction was performed in the second stage of debridement, following 15 days' negative pressure treatment (Figure 2).

Three patients don't exist ischial osteomyelitis, and the necrotic areas were clear. These patients underwent debridement and first-stage repair. All of these patients were initially prescribed cephalosporin third-generation antibiotics combined with metronidazole for anti-infection. The antibiotics were adjusted based on wound culture 

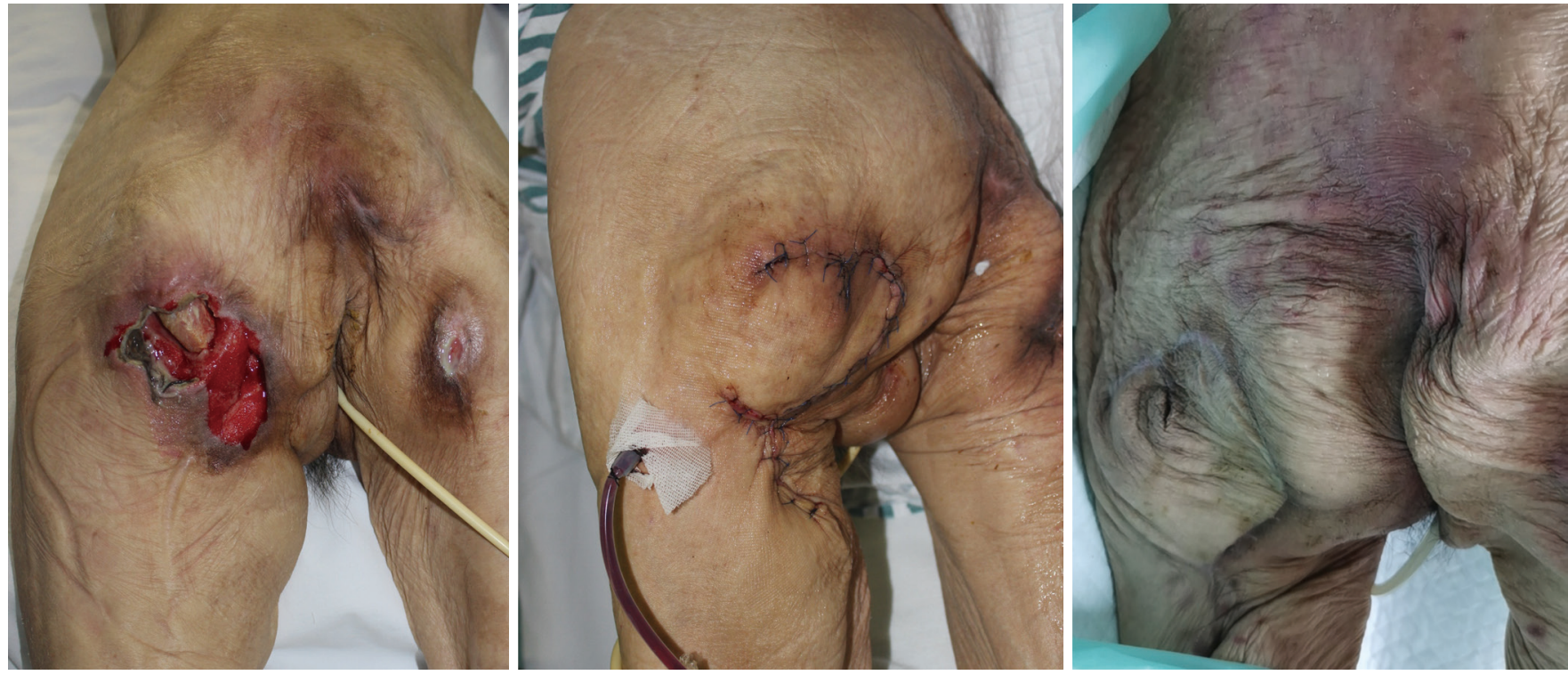

Figure 2 Reconstruction of ischial osteomyelitis with inferior gluteal artery descending branch perforator flap.

results, and sensitive antibiotics were used for at least 1 week.

All patients recovered well, and no postoperative complications were reported. During a follow-up period of 4 to 31 months, no cases of recurrence were observed. Patient information is summarized in Table 1 .

\section{Discussion}

The inferior gluteal artery descending branch perforator flap is safe and efficient for reconstructing ischial pressure sores and has exceptional advantages. Firstly, the flap is easy to design along with the surface projection of the sciatic nerve. Secondly, the procedure does not require exposure of the sciatic nerve, nor the dissection of muscle, and it has no structural influence on the buttocks or the inner side of the perineum. Therefore, if reconstruction failed, sufficient flap conditions would be preserved. Thirdly, Doppler can be used to detect whether or not a descending branch of the inferior gluteal artery exists before reconstruction, which could preserve the proximal end of the perforating vascular during the operation, with no need for precise vascular exposure. Our results showed that this flap is suitable for repairing large ischial pressure sores, but few papers had reported.

Ischial pressure sores are common in paraplegic patients who use wheelchairs. In the United States, the prevalence of ischial pressure sores in acute care hospitals, long-term care nursing facilities, and home-care settings ranges from $0.4 \%$ to $38 \%, 2 \%$ to $24 \%$, and $0 \%$ to $17 \%$, respectively (9-11). When a patient is in a sitting position, the ischial tuberosity focuses on the body support above the pelvis. This part of the body lacks muscle coverage and has only one thin skin layer and soft tissue. The pressure on the ischial region in the paraplegic patient who used a wheelchair ranges from 80 to $100 \mathrm{mmHg}$ (12). Remaining in the sitting position for a long time can lead to thrombosis of the blood vessels and ischemia and hypoxia in the local tissue (13). If this situation cannot be improved, pressure sores inevitably develop. When pressure ulcers are present, it is important to evaluate the ulcer before reconstruction. According to the pressure ulcer treatment guidelines, stage 1 and 2 ulcers can be managed conservatively; however, stage 3 and 4 ulcers generally require surgical intervention $(14,15)$.

Debridement is essential to reconstruct ischial pressure sores and prevent a recurrence. This surgery's principles are the total excision of devitalized tissues and ischial bursa, the removal of osteomyelitis bones, and the obliteration of dead spaces. Some scholars believe that prominent ischial tuberosities are prone to pressure sores. The use of an electric shovel to remove 3-4 $\mathrm{mm}$ of ischial bone, and ensuring the bone surface is flat with no bone spurs and is slightly lower than the physiological contralateral ischial bone, can effectively prevent pressure sore recurrence (16). Patients with stage 4 ischial pressure sores often have ischial osteomyelitis. In these patients, the inflammatory 


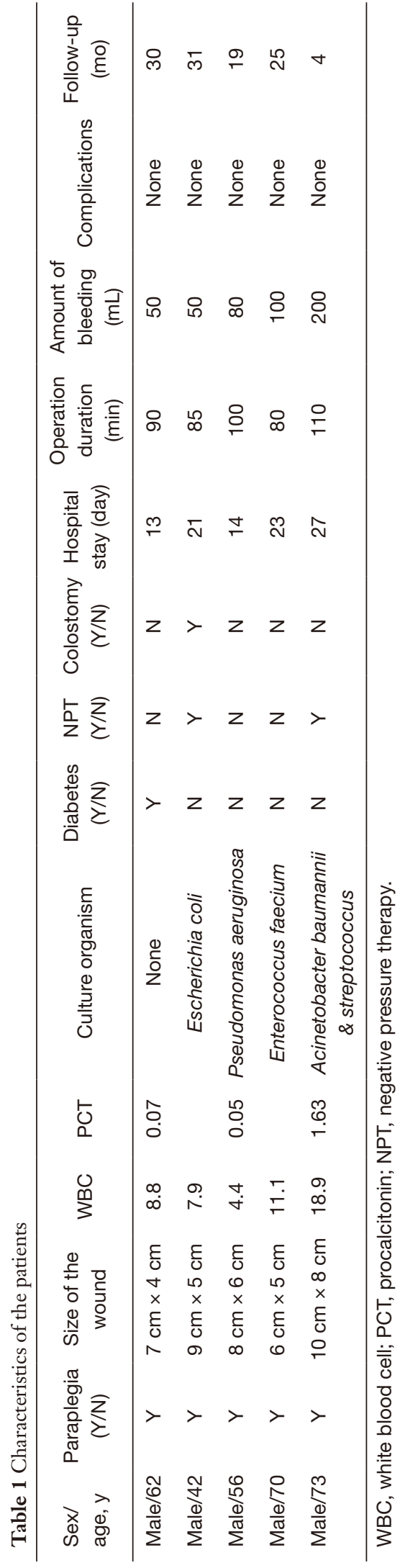

bone should be removed completely with negative pressure therapy, and then repaired in the next stages. Negative pressure therapy can effectively reduce the tissue edema of wounds, reduce the colonization rate of bacteria, increase the blood supply of local tissues, and provide good repair conditions for wounds with more serious infections (17).

The selection of a suitable repair procedure should be based on the shape and size of the wound. The shape of pressure sores is often closely related to the ischial tuberosity's anatomy; therefore, the wound is usually fusiform, diagonally inward, and downward. Moreover, these patients' gluteus usually atrophy, surrounding tissue easy to push. Therefore, if the wound is no wider than $4 \mathrm{~cm}$, reconstruction can be achieved through direct closure or a V-Y advancement flap (18). However, V-Y advancement flaps are normally used to cover small wounds, so in the repair of large defects, the distance and tension between the pedicle and ischium flaps should be considered. Despite the advantages of free flaps, the longer operative time and the procedure's complexity mean they are not the first choice for reconstruction surgery. Internal pudendal artery perforator flap has been reported to be a good method for reconstruction, but the cross-section tension on the donor site is not conducive to direct closure. Sensate anterolateral thigh perforator flap is also a good option for reconstructing ischial pressure sores, which can effectively prevent the recurrence of pressure sores (19); however, as the patients in our report had paraplegia, sensitive branches within the flap would have been worthless.

In this study, we reconstructed large ischium sore defects in five cases. In one patient, the wound was located at the edge of the scrotum and had a width of $5 \mathrm{~cm}$. An inferior gluteal artery descending branch perforator flap could easily repair this defect. Scheufler et al. also reported on inferior gluteal artery descending branch perforator flaps, centered on a perforator flap below the hip, designed to advance or rotate on the horizontal axis (20). However, our design was mainly based on the vertical axis of the thigh. The flap was dissected according to the anatomical position, and rotated to the wound at an angle of approximately $45^{\circ}$. This design will not damage the perforators of the internal pudendal artery and the perforators above the buttocks, thus without compromising future flap selection.

Some studies have suggested that filling a wound with a muscle flap has the benefit of improving local blood supply, as well as repairing wounds. However, some scholars have observed gradual muscle atrophy after transplantation. The reasons for this include muscle detachment, denervation, 
partial devascularization, and weight-bearing pressure. The prognosis is no different from flap coverage alone (21).

Our study has certain limitations. First, the sample size was not large enough. To better evaluate the efficacy of this method, a study involving more patients should be performed. Moreover, the flap rotation point could not be higher than the first perforator vascular of the inferior gluteal artery descending branch, and it is not suitable for patients combined with the gluteal ulcer.

Overall, the inferior gluteal artery descending branch perforator flap is safe and efficient for the reconstruction of ischial pressure sores. The procedure is simple and reliable, and it will produce similarly good results if applied to other patients.

\section{Conclusions}

Inferior gluteal artery descending branch perforator flap has certain advantages in the treatment of ischial pressure sores. It is easy and simple to perform, with a short operative time and minimal blood loss. Moreover, it preserves most future surgical options by reducing additional damage to the surrounding structures. Therefore, it could be used as the first-line treatment for most patients with ischial pressure sores.

\section{Acknowledgments}

Funding: This research was funded by the Medical Scientific Research Foundation of Guangdong Province of China (grant No. A2016108).

\section{Footnote}

Reporting Checklist: The authors have completed the Case Series reporting checklist. Available at http://dx.doi. org/10.21037/apm-20-1619

Data Sharing Statement: Available at http://dx.doi. org/10.21037/apm-20-1619

Conflicts of Interest: All authors have completed the ICMJE uniform disclosure form (available at http://dx.doi. org/10.21037/apm-20-1619). The authors have no conflicts of interest to declare.

Ethical Statement: The authors are accountable for all aspects of the work in ensuring that questions related to the accuracy or integrity of any part of the work are appropriately investigated and resolved. The study was conducted in accordance with the Declaration of Helsinki (as revised in 2013). The study was approved by the institutional board of Guangdong Provincial People's Hospital, Guangdong Academy of Medical Sciences (No. GDREC2019179H) and informed consent was obtained from all the patients.

Open Access Statement: This is an Open Access article distributed in accordance with the Creative Commons Attribution-NonCommercial-NoDerivs 4.0 International License (CC BY-NC-ND 4.0), which permits the noncommercial replication and distribution of the article with the strict proviso that no changes or edits are made and the original work is properly cited (including links to both the formal publication through the relevant DOI and the license). See: https://creativecommons.org/licenses/by-nc-nd/4.0/.

\section{References}

1. Kim YS, Lew DH, Roh TS, et al. Inferior gluteal artery perforator flap: a viable alternative for ischial pressure sores. J Plast Reconstr Aesthet Surg 2009;62:1347-54.

2. Legemate CM, van $\operatorname{der} \operatorname{Kwaak} M$, Gobets D, et al. The pedicled internal pudendal artery perforator (PIPAP) flap for ischial pressure sore reconstruction: Technique and long-term outcome of a cohort study. J Plast Reconstr Aesthet Surg 2018;71:889-94.

3. ScheflanM, Nahai F, Bostwick J 3rd. Gluteus maximus island musculocutaneous flap for closure of sacral and ischial ulcers. Plast Reconstr Surg 1981;68:533-8.

4. Lee SS, Huang SH, Chen MC, et al. Management of recurrent ischial pressure sore with gracilis muscle flap and $\mathrm{V}$-Y profunda femoris artery perforator-based flap. J Plast Reconstr Aesthet Surg 2009;62:1339-46.

5. Lemaire V, Boulanger K, Heymans O. Free flaps for pressure sore coverage. Ann Plast Surg 2008;60:631-4.

6. Sameem M, Au M, Wood T, et al. A systematic review of complication and recurrence rates of musculocutaneous, fasciocutaneous, and perforator-based flaps for treatment of pressure sores. Plast Reconstr Surg 2012;130:67e-77e.

7. Hurwitz DJ, Swartz WM, Mathes SJ. The gluteal thigh flap: a reliable, sensate flap for the closure of buttock and perineal wounds. Plast Reconstr Surg 1981;68:521-32.

8. Windhofer C, Brenner E, Moriggl B, et al. Relationship between the descending branch of the inferior gluteal artery and the posterior femoral cutaneous nerve applicable 
to flap surgery. Surg Radiol Anat 2002;24:253-7.

9. Lyder $\mathrm{CH}$. Pressure ulcer prevention and management. JAMA 2003;289:223-6.

10. Pressure ulcers in America: prevalence, incidence, and implications for the future. An executive summary of the National Pressure Ulcer Advisory Panel monograph. Adv Skin Wound Care 2001;14:208-15.

11. VanGilder C, Amlung S, Harrison P, et al. Results of the 2008-2009 International Pressure Ulcer Prevalence Survey and a 3-year,acute care, unit-specific analysis. Ostomy Wound Manage 2009;55:39-45.

12. Keys KA, Daniali LN, Warner KJ, et al. Multivariate predictors of failure after flap coverage of pressure ulcers. Plast Reconstr Surg 2010;125:1725-34.

13. Vohra RK, McCollum CN. Pressure sores. BMJ 1994;309:853-7.

14. Black J, Baharestani MM, Cuddigan J, et al. National Pressure Ulcer Advisory Panel's updated pressure ulcer staging system. Adv Skin Wound Care 2007;20:269-74.

15. Dini V, Bertone M, Romanelli M. Prevention and management of pressure ulcers. Dermatol Ther

Cite this article as: Li H, Lai W, Zheng S, Huang Z, Liu Z, Xiong B. Successful reconstruction of ischial pressure sores with inferior gluteal artery descending branch perforator flap. Ann Palliat Med 2021;10(4):3692-3698. doi: 10.21037/apm-20-1619
2006;19:356-64.

16. Burm JS, Hwang J, Lee YK. A New Option for the Reconstruction of Primary or Recurrent Ischial Pressure Sores: Hamstring-Adductor Magnus Muscle Advancement Flap and Direct Closure. Ann Plast Surg 2018;80:400-5.

17. Luckraz H, Murphy F, Bryant S, et al. Vacuum-assisted closure as a treatment modality for infections after cardiac surgery. J Thorac Cardiovasc Surg 2003;125:301-5.

18. Arregui J, Cannon B,Murray JE, et al. Long-term evaluation of ischiectomy in the treatment of pressure ulcers. Plast Reconstr Surg 1965;36:583-90.

19. Santanelli di Pompeo F, Longo B, Pagnoni M, et al. Sensate anterolateral thigh perforator flap for ischiatic sores reconstruction in meningomyelocele patients. Microsurgery 2015;35:279-83.

20. Scheufler O, Farhadi J, Kovach SJ, et al. Anatomical basis and clinical application of the infragluteal perforator flap. Plast Reconstr Surg 2006;118:1389-400.

21. Daniel RK, Faibisoff B. Muscle coverage of pressure points-the role of myocutaneous flaps. Ann Plast Surg 1982;8:446-52. 